\title{
Improving the Throughput of DS-CDMA Systems Using Adaptive Rate Transmissions Based on Variable Spreading Factors
}

\author{
Lie-Liang Yang and Lajos Hanzo \\ Dept. of ECS, University of Southampton, SO17 1BJ, UK. \\ Tel: +44-23-8059 3125, Fax: +44-23-8059 4508 \\ Email: 1ly,lh@ecs.soton.ac.uk; http://www-mobile.ecs.soton.ac.uk
}

\begin{abstract}
In this contribution adaptive rate transmissions are investigated in the context of DS-CDMA systems using variable spreading factors (VSF). In contrast to conventional adaptive rate transmission schemes, where the transmission rate is usually adapted in response to the channel fading, in this contribution the transmission rate is adapted in response to the multiuser interference (MUI) level. We present the philosophy of the proposed adaptive rate transmission scheme and analyze the effective throughput as well as the resultant bit error rate (BER) performance, when communicating over additive white Gaussian noise (AWGN) channels. Our study shows that when the number of active users in a DS-CDMA system can be modelled with the aid of a Markov chain and when the conventional matched filter based receiver is employed, the VSF-assisted adaptive rate transmission scheme is capable of substantially increasing the system's effective throughput. Specifically, our results show that the effective throughput may be increased by up to $40 \%$, when compared to that of DS-CDMA systems using constant spreading factors. This increased throughput is achieved without wasting power, without imposing extra interference upon other users and without increasing the BER.
\end{abstract}

\section{INTRODUCTION}

DS-CDMA is the prevalent technique in the third-generation $(3 \mathrm{G})$ wireless communications systems, because it is capable of providing numerous advantages compared to the other solutions. The capacity of DS-CDMA systems is limited by both the time-varying characteristics of the wireless channel and the multiple-access interference (MAI) or multiuser interference (MUI). The family of efficient techniques designed for compensating for the time-varying nature of the wireless channels include the popular RAKE receiver [1] contrived for achieving frequency diversity. Alternatively, multiple transmit and/or receiver antennas can be employed for achieving spatial diversity [2], [3]. The most efficient technique of combating the MAI is multiuser detection (MUD) [4]. The above techniques have attracted world-wide attention in recent years.

Another efficient technique of increasing the capacity of timevarying wireless channels is the employment of adaptive rate transmissions [5], [6], [7], where the transmission rate can be adaptively adjusted according to the instantaneous channel conditions. The main philosophy behind adaptive rate transmissions is the real-time balancing of the link budget through adaptive variation of the symbol rate, modulation constellation size and format, spreading factor, coding rate/scheme, etc, or in fact any combination of these parameters [7]. However, the results of [6] have shown that when a sufficiently high diversity order is available, regardless, whether this is due to transmitter or receiver diversity achieved in the time or frequency domain, the advantages of adaptive rate transmissions erode. Hence, in the context of the 3G DS-CDMA systems using power control, the channel fading can be efficiently mitigated by employing both the RAKE receiver and multiple transmitter/receiver antennas. In order to combat the MUI in DS-CDMA systems, as we have mentioned above, the most efficient approach is to use multiuser detection receivers [4]. The main obstacle of employing DS-CDMA MUD receivers is the high complexity of the multiuser detection algorithms. Therefore, the conventional matched filter based receiver remains popular because of its simplicity, despite its suboptimal performance.

In this contribution we consider the problem of how the effective throughput of DS-CDMA systems can be increased, when the conventional matched filter based receiver is employed. Specifically, we consider a single cell DS-CDMA system, where the number of active mobile users obeys the Poisson distribution [8] and all the signals transmitted by the mobile users are ideally power controlled. Hence, the multiuser interference level can be modelled as a discrete Markov process [8], which describes the number of active mobile users. In order to exploit the time-varying nature of the multiuser interference level, an adaptive rate transmission scheme using variable spreading factors (VSF) [9] is proposed for increasing the effective throughput. In contrast to the conventional VSF-assisted adaptive rate transmission scheme, where the transmission rate is adapted in response to the channel quality fluctuation recorded at the output of the MUD [10], the transmission rate in the proposed scheme is adapted in response to the time-varying interference level due to the MUI, while maintaining the required target BER value. In this contribution the performance of the DS-CDMA systems using the proposed VSF-assisted adaptive rate transmission scheme is evaluated when communicating over Additive White Gaussian Noise (AWGN) channels. The reasons for us to consider only AWGN channels are as follows. Firstly, as we have mentioned above, the fading effects encountered in power-controlled DS-CDMA systems can be efficiently mitigated by using RAKE receivers and multiple transmitter/receiver antennas. Secondly, our aim is to study the effects of the MUI in isolation, without the obfuscating effects of the channel's fading and then gain insight into the effects of the MUI on the system's effective throughput, when the conventional matched filter receiver is considered. Our results show that by employing VSF-assisted adaptive rate transmissions, the effective throughput of a DS-CDMA system may be increased by $40 \%$ upon exploiting the Markovian distributed number of active users in the system. The increased effective throughput is achieved without wasting power and without increasing the bit error ratio (BER).

\section{SYSTEM OVERVIEW}

We consider a single cell DS-CDMA system, where a single base station (BS) is located at the center of the cell, while the mobile users are uniformly distributed in the area covered by this base station. The based station is capable of simultaneously processing a maximum number of $K$ calls, i.e. the maximum number of active users supported by the cell is $K$. We assume that each active user's data is BPSK modulated and it is transmitted to the base station asynchronously over 
AWGN channels. Furthermore, we assume ideal power control, i.e. the received power of each active user is the same at the BS. Based on the above assumptions and assuming furthermore that there are $K_{l}+1$ active users (the reference user plus $K_{l}$ interfering users), then the received signal at the base station can be expressed as

$$
\begin{array}{r}
r(t)=n(t)+\sum_{k=1}^{K_{l}+1} \sqrt{2 P} a_{k}\left(t-\tau_{k}\right) b_{k}\left(t-\tau_{k}\right) \\
\cdot \cos \left(\omega_{c} t+\phi_{k}\right),
\end{array}
$$

where $n(t)$ is the AWGN having a two-sided spectral density of $N_{0} / 2$, $P$ represents the power received from each active user, $a_{k}(t)$ is the spreading code, $b_{k}(t)$ is the data signal, $\tau_{k}$ is the time delay parameter that accounts for the propagation delay as well as for the lack of synchronism between the transmitters, while $\phi_{k}$ is the phase angle due to carrier modulation and channel delay.

The bit errors in DS-CDMA systems communicating over AWGN channels is caused by the effect of multiple access interference and the AWGN. The BER of an asynchronous DS-CDMA system having received signals given by (1) can be closely approximated by [11]

$$
\begin{gathered}
P_{e}\left(K_{l}\right)=\frac{2}{3} Q\left[\left(\frac{K_{l}}{3 N}+\frac{1}{2 N \gamma_{c}}\right)^{-1 / 2}\right] \\
+\frac{1}{6} Q\left[\left(\frac{K_{l} N / 3+\sqrt{3} \sigma}{N^{2}}+\frac{1}{2 N \gamma_{c}}\right)^{-1 / 2}\right] \\
+\frac{1}{6} Q\left[\left(\frac{K_{l} N / 3-\sqrt{3} \sigma}{N^{2}}+\frac{1}{2 N \gamma_{c}}\right)^{-1 / 2}\right],
\end{gathered}
$$

where $\gamma_{c}=P T_{c} / N_{0}=E_{c} / N_{0}$ represents the signal to noise ratio (SNR) per chip, $T_{c}$ is the chip-duration, while the variable $N$ represents the spreading factor (number of chips per bit). In our further discourse $N$ will be controlled as a function of the number of active users and the target BER. Furthermore, the parameter $\sigma$ can be derived from the following equation [11]

$$
\sigma^{2}=\left(K_{l}+1\right)\left[N^{2} \frac{23}{360}+N\left(\frac{1}{20}+\frac{K_{l}}{36}\right)-\frac{1}{20}-\frac{K_{l}}{36}\right] .
$$

In (2) the Gaussian $Q$-function is given by $Q(x)=$ $\frac{1}{\sqrt{2 \pi}} \int_{x}^{\infty} \exp \left(-t^{2} / 2\right) d t$.

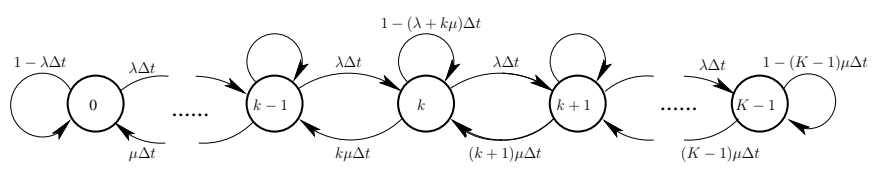

Fig. 1. State transition diagram modelling the number of active interfering users with the aid of a Markov chain having $K$ states.

The number of interfering users, $K_{l}$ can be modelled with the aid of a Markov chain having $K$ states [8]. The state transition diagram modelling the number of users determining the interference level is shown in Fig.1, which represents a $M / M / m / m$ queueing system [8]. The arrival rate of new calls or users $\lambda$ corresponds to the probability of the event that a new interfering user is activated within a unit-length timeduration, while the average service time of $1 / \mu$ is the average duration of an active interfering connection. For the $M / M / m / m$ queueing

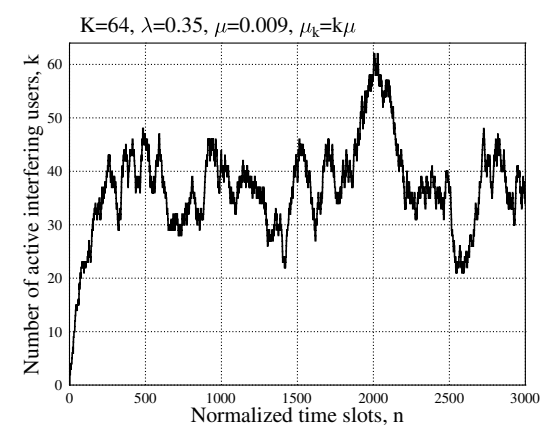

(a) Time slot interval, [0, 3000]

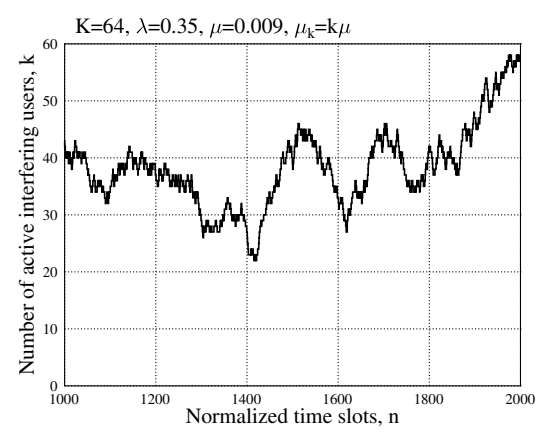

(b) Time slot interval, [1000, 2000]

Fig. 2. Markov characteristics of the number of active interfering users.

system, the probability that there are $K_{l}$ active interfering users (customers) can be expressed as [8]

$$
p_{K_{l}}=\frac{\left(\frac{\lambda}{\mu}\right)^{K_{l}} \frac{1}{K_{l} !}}{\sum_{m=0}^{K-1}\left(\frac{\lambda}{\mu}\right)^{m} \frac{1}{m !}}, K_{l}=0,1, \ldots, K-1,
$$

where the probability of simultaneously supporting $K_{l}=K-1$ users is known as the Erlang $B$ formula [8], which determines the call blocking probability of the system considered.

Fig. 2 shows number of active users generated by the abovementioned Markov chain for the first 3000 normalized time slots (Fig.2(a)) and for the normalized time slots spanning the index-range of 1000 to 2000 (Fig.2(b)). The parameters used for the simulations were $\lambda=0.35, \mu=0.009$ and the maximum number of users supported was $K=64$. From Fig.2(a) and Fig.2(b) we can observe that the number of active interfering users is a slowly time-variant variable, fluctuating as a function of the normalized time slot index.

It is widely recognized that DS-CDMA systems are interferencelimited systems and the systems' BER performance is highly sensitive to the number of interfering users. Fig.3 shows the achievable BER performance with respect to the number of active users for a DS-CDMA system communicating over AWGN channels, when 


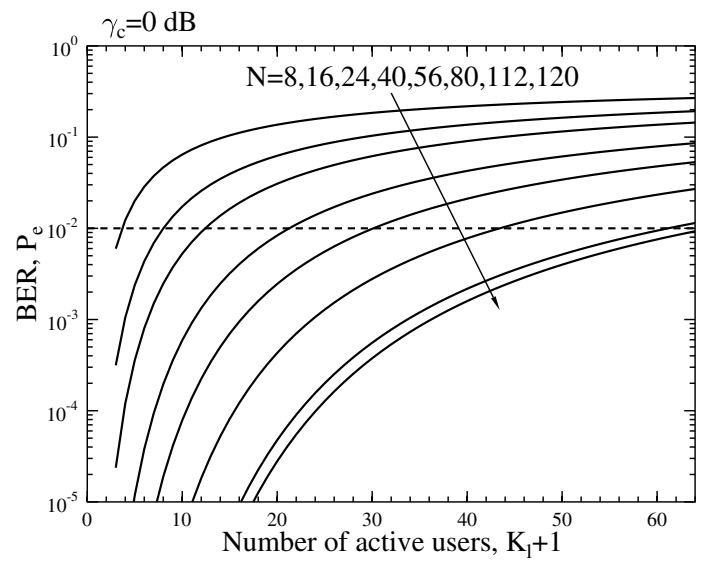

Fig. 3. BER performance versus the number of active users for the parameters of $\gamma_{c}=0 \mathrm{~d} B$ and spreading factors of $N=8,16,24,40,56,80,112,120$ computed from (2).

the spreading factors of $N=8,16,24,40,56,80,112,120$ are employed. From the results of Fig. 3 we can infer following observations.

- For a given spreading factor, the BER increases when supporting an increased number of active users;

- For a given number of users, the BER decreases upon increasing the value of the spreading factor;

- For a given target BER - for example for $P_{E}=10^{-2}$ - and for a given number of active users, there exists a spreading factor, which results in a specific BER for the DS-CDMA system matching the target BER requirement. A DS-CDMA system using a higher spreading factor is capable of supporting a higher number of active users, than that using a lower spreading factor, while maintaining the target BER.

Therefore, based on the Markov chain characterized in Fig.2 and on the BER performance of the DS-CDMA system as a function of the number of active users shown in Fig.3, we argue that an appropriate spreading factor can be employed within a specific time slot for maximizing the number of bits transmitted by this specific time slot, while maintaining the required BER performance. Furthermore, when the number of active users dynamically fluctuates, variable spreading factors can be employed by the DS-CDMA system for achieving the maximum throughput, while guaranteeing the required BER performance. In other words, VSF-assisted adaptive DS-CDMA system is capable of increasing the effective throughput of the system, while maintaining a given target BER.

\section{AdAPtive Transmission Scheme}

In this section we propose and investigate a adaptive rate transmission scheme based on VSF. Let us assume that each user transmits a block of data as shown in Fig.4. As shown in Fig.4, the data block is divided into $L$ frames. We assume that at the beginning of the $l$ th frame there are $K_{l}$ interfering users, which is the a priori knowledge for determining the required spreading factor and the corresponding transmission rate during the $l$ th frame. Let us assume that each frame consists of a constant number of chips, which is expressed as $N_{f}$, i.e. each frame has a constant duration of $N_{f} T_{c}$ seconds. Let us also assume that there are $m$ spreading factors having values expressed as $N_{1}<N_{2}<\ldots<N_{m}$, where each of them is a multiple of $N_{f}$. Furthermore, let us assume that the target BER is $P_{E}$. Then the required spreading factor of the $l$ th frame and the corresponding number of bits conveyed by the $l$ th frame can be determined as follows.

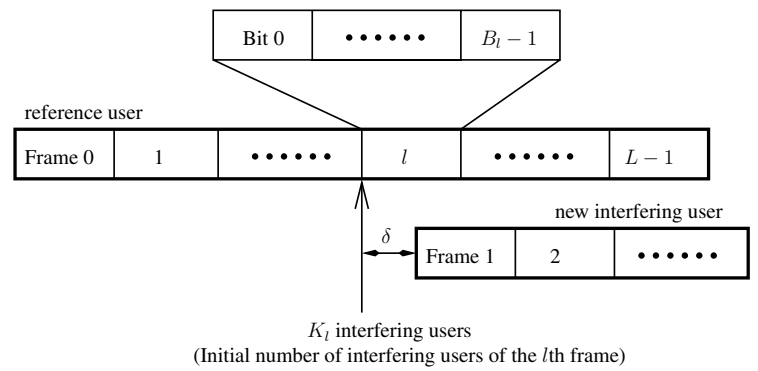

Fig. 4. Data structure of the transmitted signal in adaptive rate DS-CDMA systems using VSF-assisted adaptive rate transmissions.

- A specific spreading factor is selected from the set $\left\{N_{1}, N_{2}, \ldots, N_{m}\right\}$ according to Equation (2) based on the number of interfering users, $K_{l}$, and on the target BER, $P_{E}$, such that the selected spreading factor's value is as low as possible, while guaranteeing the required BER performance. We denote the selected spreading factor by $N_{l}\left(K_{l}, P_{E}\right)$.

- Once the spreading factor, $N_{l}\left(K_{l}, P_{E}\right)$ of the $l$ th frame was selected, the corresponding number of bits, $B_{l}\left(K_{l}, P_{E}\right)$, conveyed by the $l$ th frame can be determined as

$$
B_{l}\left(K_{l}, P_{E}\right)=\frac{N_{f}}{N_{l}\left(K_{l}, P_{E}\right)} .
$$

In [5] Goldsmith and Chua have shown that the highest effective throughput can be achieved by using continuous rate adaptation. Hence, the results of [5] suggest that we should use as many different spreading factors as possible and, simultaneously, have a nearcontinuous spreading factors value set. Table I shows the design example for the appropriate choice of the spreading factors. In Table I we assumed that random spreading sequences having various length were employed, and that the frame's length is $N_{f}=2^{4} \times 3 \times 5 \times 7=1680$ chips. As shown in Table I, we can obtain 40 different spreading factors and hence can support 40 different transmission rates.

\section{Throughput AND BER ANALYSIS}

In this section we analyze the effective throughput as well as the resultant average BER, when achieving this effective throughput. The effective throughput is defined as the total number of bits successfully transmitted within a unity-duration time-interval by all users supported by the system. Our analysis is based on the following assumptions. (a) All active users communicate using adaptive rate transmissions based on the same set of spreading factors, as described in Section III. The transmitted data block-length of each active user obeys an independent identical distribution (i.i.d). (b) Assuming that the number of interfering users at the beginning of the $l$ th frame is $K_{l}$, the probability of increasing or decreasing this number by one within a frame's time duration is given by $P_{K_{l}+}=\lambda$ or $P_{K_{l}-}=K_{l} \mu$, respectively. The probability of increasing or decreasing the number of interfering users 


\begin{tabular}{|c|c|c||c|c|c|}
\hline Index & Bits/frame & Spreading factors & Index & Bits/frame & Spreading factors \\
\hline 1 & 1 & 1680 & 21 & 42 & 40 \\
2 & 2 & 840 & 22 & 48 & 35 \\
3 & 3 & 560 & 23 & 56 & 30 \\
4 & 4 & 420 & 24 & 60 & 28 \\
5 & 5 & 336 & 25 & 70 & 24 \\
6 & 6 & 280 & 26 & 80 & 21 \\
7 & 7 & 240 & 27 & 84 & 20 \\
8 & 8 & 210 & 28 & 105 & 16 \\
9 & 10 & 168 & 29 & 112 & 15 \\
10 & 12 & 140 & 30 & 120 & 14 \\
11 & 14 & 112 & 31 & 140 & 12 \\
12 & 15 & 105 & 32 & 168 & 10 \\
13 & 16 & 84 & 33 & 210 & 8 \\
14 & 20 & 80 & 35 & 240 & 7 \\
15 & 21 & 70 & 36 & 336 & 6 \\
16 & 24 & 60 & 37 & 420 & 5 \\
17 & 28 & 56 & 38 & 560 & 4 \\
18 & 30 & 48 & 39 & 840 & 3 \\
19 & 35 & 42 & 40 & 1680 & 2 \\
20 & 40 & & & & 1 \\
\hline
\end{tabular}

TABLE I

NUMBER OF BITS TRANSMITTED IN A FRAME By ASSUMING THAT THE TOTAL NUMBER OF CHIPS PER FRAME IS $N_{f}=1680=2^{4} \times 3 \times 5 \times 7$ WHEN THE SPREADING FACTORS SEEN IN THE RIGHT COLUMN ARE EMPLOYED.

within a frame's time duration by more than one is zero. Therefore, the probability that the number of interfering users remains unchanged, i.e. $K_{l}$, within a frame's time duration can be expressed as

$$
P_{K_{l}}= \begin{cases}1-\lambda & \text { if } K_{l}=0 \\ 1-\lambda-K_{l} \mu & \text { if } 1 \leq K_{l} \leq K-2 \\ 1-K_{l} \mu & \text { if } K_{l}=K-1\end{cases}
$$

(c) When the number of active interfering users increases by one or decreases by one within the $l$ th frame, we assume that this happens at the moment having a time difference of $\delta$ from the beginning of the $l$ th frame (see Fig.4), where $\delta$ is assumed to be uniformly distributed over the interval $\left[0, N_{f} T_{c}\right)$.

Based on the above assumptions, the effective throughput can be derived and is expressed as

$$
\begin{aligned}
B= & \sum_{K_{l}=0}^{K-1} \frac{\left(\frac{\lambda}{\mu}\right)^{K_{l}} \frac{1}{K_{l} !}}{\sum_{m=0}^{K-1}\left(\frac{\lambda}{\mu}\right)^{m} \frac{1}{m !}}\left[\frac{K_{l}+1}{N_{l}\left(K_{l}, P_{E}\right)}\right. \\
& \left.+\left(\lambda-K_{l} \mu\right) \times \frac{1}{2 N_{l}\left(K_{l}, P_{E}\right)}\right] \\
& -\frac{\left(\frac{\lambda}{\mu}\right)^{K-1} \frac{1}{(K-1) !}}{\sum_{m=0}^{K-1}\left(\frac{\lambda}{\mu}\right)^{m} \frac{1}{m !}} \times \frac{\lambda}{2 N_{l}\left(K-1, P_{E}\right)} \text { [bits/chip].}
\end{aligned}
$$

The average BER, when the effective throughput of (7) has been achieved, can be expressed as

$$
\begin{aligned}
& P_{e}=\sum_{K_{l}=0}^{K-1} p_{K_{l}} \hat{P}_{e}\left(K_{l}\right) \\
& =\sum_{K_{l}=0}^{K-1} \frac{\left(\frac{\lambda}{\mu}\right)^{K_{l}} \frac{1}{K_{l} !}}{\sum_{m=0}^{K-1}\left(\frac{\lambda}{\mu}\right)^{m} \frac{1}{m !}} \times\left(\frac{\lambda}{2}\left[P_{e}\left(K_{l}\right)+P_{e}\left(K_{l}+1\right)\right]\right.
\end{aligned}
$$

$$
\begin{aligned}
+\frac{K_{l} \mu}{2}[ & \left.\left.P_{e}\left(K_{l}\right)+P_{e}\left(K_{l}-1\right)\right]+\left(1-\lambda-K_{l} \mu\right) P_{e}\left(K_{l}\right)\right) \\
& -\frac{\left(\frac{\lambda}{\mu}\right)^{K-1} \frac{1}{(K-1) !}}{\sum_{m=0}^{K-1}\left(\frac{\lambda}{\mu}\right)^{m} \frac{1}{m !}} \\
& \times\left(\frac{\lambda}{2}\left[P_{e}(K-1)+P_{e}(K)\right]-\lambda P_{e}(K-1)\right) .
\end{aligned}
$$

Note that in (8) $P_{e}\left(K_{l}\right), P_{e}\left(K_{l}+1\right)$ and $P_{e}\left(K_{l}-1\right)$ are functions of $N_{l}\left(K_{l}, P_{E}\right)$, since the spreading factor assumes different values in response to the time-variant number of active interfering users in the context of the VSF-assisted adaptive rate transmission scheme.

\section{Numerical Results AND Discussions}

In this section we provide some performance results, in order to compare the throughput performance of the proposed adaptive rate and that of the conventional constant rate DS-CDMA transmission scheme. The spreading factors employed for adaptive rate transmissions are shown in Table I. Furthermore, in the context of the DS-CDMA system using constant rate transmissions we assumed that at any given SNR/chip value the specific spreading factor was used, which was capable of maximizing the effective throughput, while guaranteeing the target BER performance, for the given distribution of the number of interfering users.

In Fig. 5 we compared the throughput versus SNR/chip performance of the DS-CDMA system using VSF-assisted adaptive transmissions to that of the DS-CDMA system using constant rate transmissions, when various call generation rates and average call durations are considered. The results of Fig.5 show that the adaptive rate transmission scheme significantly outperforms the constant rate transmission scheme. Specifically, the adaptive rate transmission scheme is capable of providing an approximately $40 \%$ higher effective throughput than the constant rate transmission scheme. The plausible justification for these results is that when adaptive rate transmission is employed, 


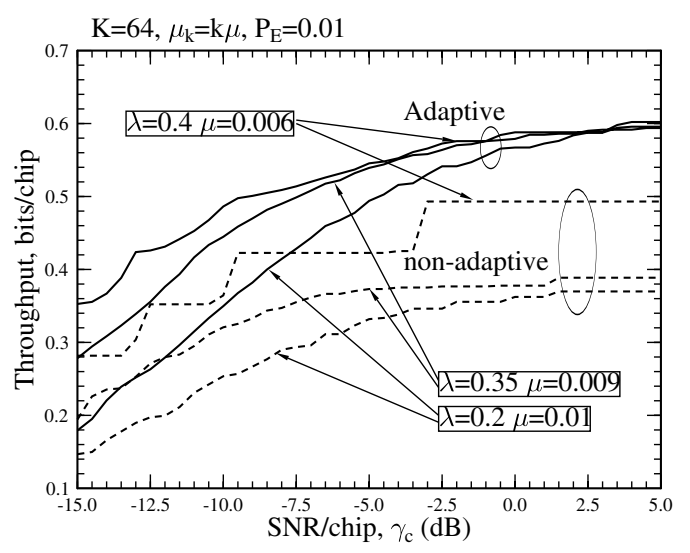

Fig. 5. Throughput performance comparison of the constant spreading factor assisted non-adaptive DS-CDMA scheme and the VSF-assisted adaptive DSCDMA arrangement.

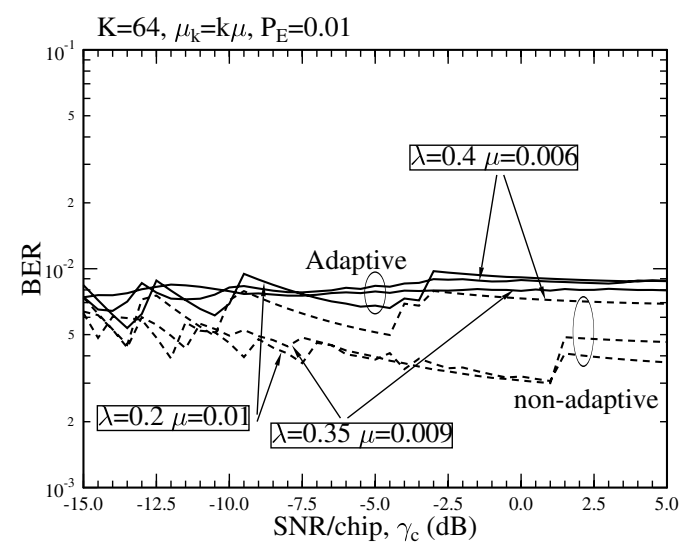

Fig. 6. BER performance comparison between the constant spreading factor assisted non-adaptive DS-CDMA and the VSF-assisted adaptive DS-CDMA schemes, when they achieve the effective throughputs of Fig.5.

the system is capable of accommodating the interference level experienced by activating an appropriate spreading factor according to the number of active interfering users at a given SNR/chip value. By contrast, for the constant rate transmission scheme, only single spreading factor is employed at a given SNR/chip value, regardless of the number of users supported. Consequently, when the number of active interfering users is low, the BER performance will be better than the target BER and hence the effective throughput is correspondingly lower than necessary. However, when the number of active interfering users is excessive, the BER of the received data is higher than the target BER. Hence the received data may have to be discarded without contributing to the effective throughput.

Fig.6 we show the resultant BER performance of the DS-CDMA system using both constant rate transmissions and the proposed adaptive rate transmission scheme, when they achieve the effective through- put values shown in Fig.5. From the results of Fig.6 we observe that for each group of $(\lambda, \mu)$ values, the constant rate scheme has a lower BER, than the adaptive rate scheme, while the BER of the adaptive rate transmission scheme is closer to the target BER, than that of the constant rate transmission scheme. The results of Fig.6 demonstrate that neither the BER of the constant rate nor that of the adaptive rate transmission scheme fluctuates dramatically. Indeed, they both remain in the BER range of $(0.001,0.01)$ for various of the SNR/chip value. The reason for observing a near-constant BER even for the constant rate transmission scheme is because we deliberately adjusted the data rate in response to the SNR/chip value experienced for the sake of fair comparison, although a practical constant-rate system is incapable of doing so. By contrast, the adaptive rate transmission scheme was capable of adapting the data rate in response to both the SNR/chip value as well as the number of active interfering user supported at each specific SNR/chip value.

In conclusions, we have shown in this contribution that, when the number of active users in a DS-CDMA system can be modelled with the aid of a Markov chain and when the conventional matched filter based receiver is employed, an adaptive rate transmission scheme using variable spreading factors (VSF) can be employed in response to the time-varying MUI level experienced. The VSF-assisted adaptive rate transmission scheme is capable of significantly increasing the system's effective throughput.

\section{REFERENCES}

[1] G. L. Turin, "Introduction to spread-spectrum antimultipath techniques and their application to urban digital radio," Proceedings of IEEE, vol. 68, pp. 328-353, March 1980.

[2] P. M. S. Affes, "A new receiver structure for asynchronous CDMA: STAR - the spatio-temporal array-receiver," IEEE Journal on Selected Areas in Communications, vol. 16, pp. 1411 - 1422, October 1998.

[3] B. Hochwald, T. L. Marzetta, and C. B. Papadias, "A transmitter diversity scheme for wideband CDMA systems based on space-time spreading," IEEE Journal on Selected Areas in Communications, vol. 19, pp. 48-60, January 2001.

[4] S. Verdu, Multiuser Detection. Cambridge University Press, 1998.

[5] A. J. Goldsmith and S. G. Chua, "Variable-rate variable-power MQAM for fading channels," IEEE Transactions on Communications, vol. 45, pp. 1218-1230, October 1997.

[6] M. S. Alouini and A. J. Goldsmith, "Capacity of Rayleigh fading channels under different adaptive transmission and diversity-combining techniques," IEEE Transactions on Vehicular Technology, vol. 48, pp. 11651181, July 1999.

[7] L.-L. Yang and L. Hanzo, "Software defined radio assisted adaptive broadband frequency hopping multicarrier DS-CDMA," To appear in IEEE Communications Magazine, vol. 40, March 2002.

[8] D. Bertsekas and R. Gallager, Data Networks. Englewood Cliffs, N.J. : Prentice Hall, 2nd ed., 1992.

[9] F. Adachi, M. Sawahashi, and H. Suda, "Wideband DS-CDMA for next-generation mobile communications systems," IEEE Communications Magazine, vol. 36, pp. 56-69, September 1998.

[10] E. L. Kuan and L. Hanzo, "Comparative study of adaptive-rate CDMA transmission employing joint-detection and interference cancellation receivers," in Proceedings of IEEE VTC'2000, (TOKYO, JAPAN), pp. 7175, May 2000.

[11] J. M. Holtzman, "A simple, accurate method to calculate spread-spectrum multiple-access error probabilities," IEEE Transactions on Communications, vol. 40, pp. 461-464, March 1992. 